\title{
Laboratory investigations of herpes simplex virus-1 and -2 clinical samples in Korea
}

\author{
Eun Ju Oh${ }^{1 \oplus}$, Young Sam Yuk ${ }^{2}{ }^{\oplus}$, Jae Kyung Kim ${ }^{3}{ }^{\oplus}$ \\ ${ }^{1}$ Department of Medical Laser, Dankook University Graduate School of Medicine, Cheonan, Korea \\ ${ }^{2}$ Department of Health Administration, Dankook University College of Health Sciences, Cheonan, Korea \\ ${ }^{3}$ Department of Biomedical Laboratory Science, Dankook University College of Health Sciences, Cheonan, Korea
}

Received: June 8, 2021

Revised: September 6, 2021

Accepted: November 15, 2021

Corresponding author:

Jae Kyung Kim

Department of Biomedical Laboratory Science, Dankook University College of Health Sciences, Cheonan, Korea E-mail: nerowolf2@dankook.ac.kr

Eun Ju Oh and Young Sam Yuk contributed equally to this work as co-first authors.

\section{ABSTRACT}

Objectives: Herpes simplex virus (HSV) infections have been reported in 60\% to $95 \%$ of the adult population worldwide, making them one of the most common infectious conditions globally. HSV-1 and HSV-2 cause severe disease in immunocompromised patients. Therefore, the aim of this study was to provide information that could be used to reduce the incidence of genital herpes caused by HSV-1 and HSV-2.

Methods: From September 2018 to December 2020, 59,381 specimens were collected from outpatients across primary and secondary hospitals in Korea who requested U2Bio (Korea) to conduct molecular biological testing of their samples for sexually transmitted infections. In this study, the positivity rates of HSV-1 and HSV-2 were analyzed according to age, sex, and specimen type.

Results: In the age-specific analysis of HSV-1, the highest positivity rate $(0.58 \%)$ was observed in patients under 19 years of age, whereas the lowest positivity rate $(0.08 \%)$ was observed in patients aged over 70 years. In the age-specific analysis of HSV-2, the highest positivity rate (2.53\%) was likewise observed in patients under 19 years of age.

Conclusion: Our study identified differences in the infection rates of HSV-1 and HSV-2 depending on patients' sex and age. These differences will be useful for improving disease prevention and control measures for HSV-1 and HSV-2.

Keywords: Human herpesvirus 1; Human herpesvirus 2; Korea; Sexually transmitted diseases

\section{Introduction}

Herpes simplex virus (HSV) infections are among the most widespread infectious conditions worldwide, affecting $60 \%$ to $95 \%$ of the adult population [1]. HSV is the most common sexually transmitted infection (STI) in the United States [2]. According to Burn Aschner et al. [3], 67\% of the worldwide population is infected with HSV-1 by 49 years of age; many individuals in 
developing countries are infected early in childhood [3].

In the United States, between 2015 and 2016, the prevalence of HSV-1 and HSV-2 among people between 14 and 49 years of age was $47.8 \%$ and $11.9 \%$, respectively [4]. During the period from October 2014 to March 2015 in Ghana, the prevalence rates of HSV-1 and HSV-2 among females were $99.2 \%$ and $78.4 \%$, respectively [5]. These results indicate that the global spread of HSV-1 and HSV-2 is high and attention-worthy [6] .

HSV-1 and HSV-2, which are double-stranded DNA viruses that typically infect humans [7], are related to many diseases and can cause rare but severe after-effects, such as meningitis and neonatal herpes [8]. Moreover, HSV infection during pregnancy is related to miscarriage, premature birth, and congenital and neonatal herpes, and it can cause severe infections in newborns owing to intrauterine vertical metastasis. In many cases, contact with HSV-infected genital secretions can lead to a high risk of mortality and neurodevelopmental disorders during vaginal delivery [1].

HSV infection can also function as a co-factor for the acquisition and transmission of other sexually contagious infections, including human immunodeficiency virus, syphilis, hepatitis C virus, and human papillomavirus [9]. HSV-1 and HSV-2 also cause blindness and severe infectious diseases in immunocompromised patients [10]. In the United States, the proportion of individuals co-infected with HSV-1 and HSV2 decreased from $14.6 \%$ in 1988-1994 to $10.5 \%$ in 1999-2004 [11]. Co-infection with HSV-1 and HSV-2 severely affects the dynamics of patients with clinical genital herpes compared with infection with HSV-2 only [12].

Vaccines that prevent genital herpes infections affect public health [13]. However, vaccines intended to prevent HSV-2 genital herpes have not been successful to date [13]. Therefore, the aim of this study was to provide information to support the development of vaccines that would be useful for protection against genital herpes caused by HSV1 and HSV-2 and to provide information that could be used to safeguard public health and reduce the incidence of genital herpes caused by HSV-1 and HSV-2.

\section{Materials and Methods}

\section{Materials}

From September 2018 to December 2020, 59,381 specimens were collected from outpatients across primary and secondary hospitals in Korea who requested U2Bio (Seoul, Korea) to conduct molecular biological testing of their samples for STIs. The specimens were classified into swab, urine, and other (catheter, pus, and tissue) samples. The study protocol was approved by the Institutional Review Board of Dankook University (IRB No. 2021-04-002).

\section{Nucleic Acid Extraction}

The collected clinical specimens were stored at $-70^{\circ} \mathrm{C}$ until DNA isolation for multiplex polymerase chain reaction (mPCR). DNA for the mPCR assay was extracted using an ExiPrep Dx Bacteria Genomic DNA kit (Bioneer, Daejeon, Korea) according to the manufacturer's instructions. The concentrations of the extracted DNA samples were measured using the AccuPower STI8B-Plex Real-Time PCR kit (Bioneer).

\section{Real-Time PCR Analysis}

Real-time PCR analysis was performed using the AccuPower STI8B-Plex Real-Time PCR kit with an Exicycler 96 RealTime Quantitative Thermal Block (Exicycler 96; Bioneer), according to the manufacturer's protocol. The amplification protocol comprised 1 cycle at $95^{\circ} \mathrm{C}$ for 5 minutes and 45 cycles at $95^{\circ} \mathrm{C}$ for 5 seconds and $55^{\circ} \mathrm{C}$ for 5 seconds. The threshold cycle was determined according to the manufacturer's instructions. Four pathogens-Trichomonas vaginalis, Mycoplasma hominis, HSV-1, and HSV-2-were evaluated. In this study, we analyzed HSV-1 and HSV-2. The real-time PCR target genes used and their product sizes are listed in Table 1.

\section{Statistical Analysis}

SAS ver. 9.4 (SAS Institute Inc., Cary, NC, USA) was used to perform all statistical analyses, including descriptive statistical analysis and frequency analysis. Statistical significance was set at $p<0.05$.

\section{Results}

In total, 59,381 specimens were tested for HSV-1 and HSV2 infections between August 2018 and December 2020, and 1,429 (2.4\%) of them tested positive.

In the age-specific analysis of HSV-1, the highest positivity rate $(0.58 \%, 6 / 1,026)$ was observed in patients under 19 years of age, followed by those aged 20 to 29 years $(0.50 \%, 80 / 16,076)$ (Figure 1). The lowest HSV-1 positivity rate $(0.08 \%, 2 / 2,370)$ was observed in patients aged over 70 years (Figure 1). In the agespecific analysis of HSV-2, the highest positivity rate $(2.53 \%$, $26 / 1,026)$ was observed in patients under 19 years of age, followed by those aged 20 to 29 years $(2.33 \%, 375 / 16,076)$. The lowest HSV-2-positivity rate $(1.76 \%, 196 / 11,119)$ was observed in patients aged 40 to 49 years (Figure 1 ). The positivity rate

Table 1. Target genes and their product sizes for real-time polymerase chain reaction

\begin{tabular}{lcc}
\hline Pathogen & Target gene & Product size (bp) \\
\hline Herpes simplex virus type 1 & us 4 & 111 \\
Herpes simplex virus type 2 & Gg & 86 \\
\hline
\end{tabular}




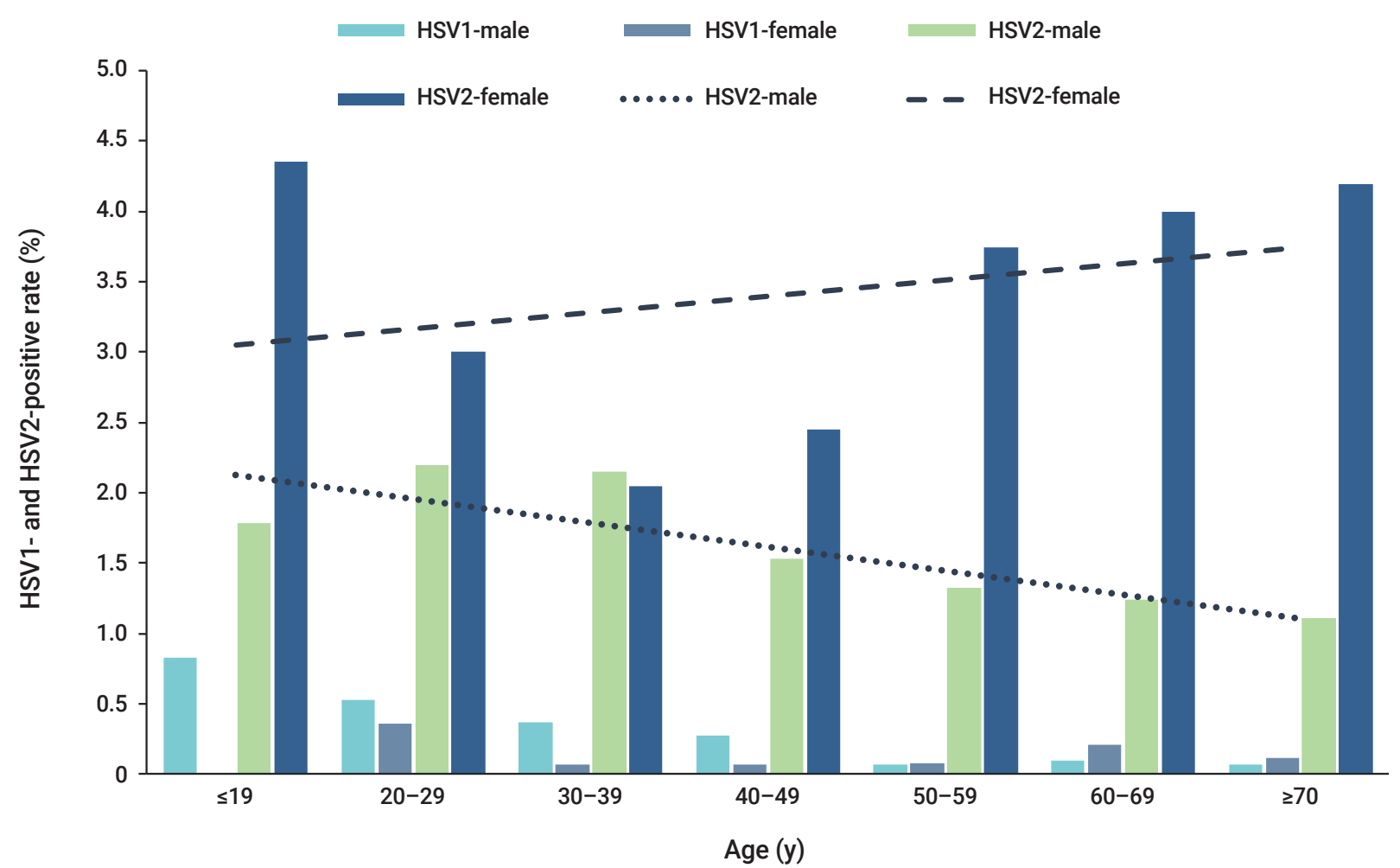

Figure 1. Herpes simplex virus (HSV)-1 and HSV-2 positivity rates according to age and sex.

The HSV-2 positivity rate was higher than the HSV-1 positivity rate. The positivity rate of HSV was higher in females than in males.

of HSV-1 showed a significant association with age $(p=0.007)$ (Table 2). Similarly, the positivity rate of HSV-2 was also significantly associated with age $(p=0.026)$ (Table 2$)$. Distinct patterns of infection were observed in different age groups.

The results of the specimen-specific positivity rate analysis showed that in males, the highest HSV-1-positivity rate was found in urine specimens $(0.66 \%, 5 / 760)$, and the highest HSV-2-positivity rate was found in swab specimens (5.92\%, 45/760) (Table 3). In females, the highest HSV-1-positivity rate was found in swab specimens $(0.23 \%, 15 / 6,665)$, and the highest HSV-2-positivity rate was found in swab specimens (3.81\%, 254/6,665) (Table 3). To summarize, the highest positivity rate was observed in swab specimens of both males and females. The HSV-2-positivity rate was higher than that of HSV-1 during the study period. HSV-1 and HSV-2 co-infection $(n=5 / 59,381)$ was observed.

\section{Discussion}

The study analyzed the positivity rates of HSV-1 and HSV2 infections according to age, sex, and specimen type from September 2018 to December 2020. The highest positivity rates for both HSV-1 and HSV-2 were observed in the specimens of patients under 19 years of age. The HSV-2 positivity rate
Table 2. Age distribution of the positivity rate of HSV-1 and HSV-2 and correlation between age and the positivity rate of HSV-1 and HSV-2 between 2018 and 2020

\begin{tabular}{lll}
\hline Age $(\mathbf{y})$ & \multicolumn{1}{c}{ HSV-1 } & HSV-2 \\
\hline$\leq 19(n=1,026)$ & $6(0.58)$ & $26(2.53)$ \\
$20-29(n=16,076)$ & $80(0.50)$ & $375(2.33)$ \\
$30-39(n=15,644)$ & $49(0.31)$ & $333(2.13)$ \\
$40-49(n=11,119)$ & $25(0.22)$ & $196(1.76)$ \\
$50-59(n=8,474)$ & $6(0.07)$ & $175(2.07)$ \\
$60-69(n=4,672)$ & $6(0.13)$ & $98(2.10)$ \\
$\geq 70(n=2,370)$ & $2(0.08)$ & $52(2.19)$ \\
$p$ & $0.007^{\star}$ & $0.026^{\star}$ \\
\hline
\end{tabular}

Data are presented as $n(\%)$.

HSV, herpes simplex virus.

${ }^{*} p<0.05$.

was higher in females than in males.

HSV-1 prevalence tends to increase with age, and most incident cases occur in childhood and adolescence. Moreover, the shapes of age-specific HSV-2 and HSV-1 prevalence graphs differ primarily in terms of whether they show the expected patterns of an STI (HSV-2) rather than a non-STI (HSV-1) [8]. The prevalence of HSV-2 was previously reported to range from $34 \%$ to $79 \%$ among clinic- and community-based 15 - 
Table 3. Positivity rates of HSV-1 and HSV-2 according to the specimen type and sex between 2018 and 2020

\begin{tabular}{|c|c|c|c|c|c|c|}
\hline \multirow{2}{*}{ Specimen } & \multicolumn{3}{|c|}{ Male } & \multicolumn{3}{|c|}{ Female } \\
\hline & Total (n) & HSV-1 & HSV-2 & Total (n) & HSV-1 & HSV-2 \\
\hline Swab & 760 & $5(0.66)$ & $45(5.92)$ & 6,665 & $15(0.23)$ & $254(3.81)$ \\
\hline Urine & 38,905 & $127(0.33)$ & $699(1.80)$ & 5,986 & $5(0.08)$ & $145(2.42)$ \\
\hline Other & 6,168 & $22(0.36)$ & $99(1.61)$ & 897 & 0 & $13(1.45)$ \\
\hline Total & 45,833 & $154(0.34)$ & $843(1.84)$ & 13,548 & $20(0.15)$ & $412(3.04)$ \\
\hline
\end{tabular}

Data are presented as $n(\%)$.

HSV, herpes simplex virus.

to 24-year-old individuals in Eastern Africa and from $46 \%$ to $66 \%$ in high-risk populations [14,15].

Sexually acquired genital HSV-1 infections are common in young females and males with active sex lives [16]. According to Issakwisa et al. [16], the infection rates are higher in males than in females [16]. Our study also showed that HSV-1 infection was more common in males than in females. The age-specific HSV-2 prevalence is usually higher in females than in males, as well as in individuals with high-risk sexual behaviors [14,17]. This result appears to be consistent across multiple geographic sites, and females seem to show a higher risk of HSV-2 acquisition than males [8].

Severe HSV-1 and HSV-2 co-infections, with high mortality and morbidity rates, have been observed in newborns [18]. HSV-1 and HSV-2 co-infection were observed in this study; however, further research is needed to verify this finding.

This study has some limitations. First, it was conducted in the tests performed by a single organization, and it did not represent all regions in Korea. Additionally, the 3-year study period was relatively short, making it difficult to identify trends in HSV-1 and HSV-2 infections. Second, the sample data were anonymous, and the residence of the patients was not specified. Third, it was a retrospective study rather than a prospective study, and the results were not analyzed according to patients' clinical characteristics.

The prevalence of HSV-2 and HSV-1 is highly variable, and it depends on many factors, including country, area of residence, population subgroup, sex, and age [10]. Therefore, age-specific or age-adjusted prevalence among similar populations should be analyzed to compare the prevalence of HSV infections across geographic areas or between countries. Despite these limitations, we found distinct infection patterns in different age groups. We expect that this information will assist in the development and implementation of targeted behavioral interventions to reduce genital herpes infections caused by HSV-1 and HSV-2.

\section{Conclusion}

Our study identified differences in the infection rates of HSV-
1 and HSV-2 according to sex and age. The characterization of these differences will be useful for describing the dynamics and risk factors of HSV-1 and HSV-2, especially for improving disease prevention and control measures. We also expect that this study will provide information that can be used to safeguard public health and reduce the incidence of genital herpes caused by HSV-1 and HSV-2.

\section{Notes}

\section{Ethics Approval}

This study was approved by the Dankook University Institutional Review Board (IRB No. 2021-04-002).

\section{Conflicts of Interest}

The authors have no conflicts of interest to declare.

\section{Funding}

None.

\section{Availability of Data}

The datasets are not publicly available but are available from the corresponding author upon reasonable request.

\section{Authors' Contributions}

Conceptualization: all authors; Data curation: EJO; Formal analysis: YSY; Investigation: all authors; Methodology: all authors; Project administration: JKK; Resources: all authors; Software: all authors; Supervision: JKK; Validation: all authors; Visualization: all author; Writing-original draft: EJO, JKK; Writing-review $\&$ editing: all authors.

\section{References}

1. Marchi S, Trombetta CM, Gasparini R, et al. Epidemiology of herpes simplex virus type 1 and 2 in Italy: a seroprevalence study from 2000 to 2014. J Prev Med Hyg 2017;58:E27-33.

2. Roberts CM, Pfister JR, Spear SJ. Increasing proportion of herpes simplex virus type 1 as a cause of genital herpes infection in college students. Sex Transm Dis 2003;30:797-800.

3. Burn Aschner C, Knipe DM, Herold BC. Model of vaccine efficacy against HSV-2 superinfection of HSV-1 seropositive mice demonstrates protection by antibodies mediating cellular cytotoxicity. NPJ Vaccines 2020;5:35.

4. McQuillan G, Kruszon-Moran D, Flagg EW, et al. Prevalence of herpes simplex virus type 1 and type 2 in persons aged 14-49: United States, 2015-2016. NCHS Data Brief 2018;(304):1-8. 
5. Debrah O, Agyemang-Yeboah F, Asmah RH, et al. SERO-prevalence of herpes simplex virus type 1 and type 2 among women attending routine Cervicare clinics in Ghana. BMC Infect Dis 2018:18:378.

6. Looker KJ, Magaret AS, May MT, et al. Global and regional estimates of prevalent and incident herpes simplex virus type 1 infections in 2012. PLoS One 2015;10:e0140765

7. Koganti R, Yadavalli T, Shukla D. Current and emerging therapies for ocular herpes simplex virus type-1 infections. Microorganisms 2019;7:429.

8. Smith JS, Robinson NJ. Age-specific prevalence of infection with herpes simplex virus types 2 and 1: a global review. J Infect Dis 2002;186 Suppl 1:S3-28.

9. Agyemang-Yeboah F, Debrah O, Timmy-Donkoh E, et al. Co-infection prevalence of herpes simplex virus types 1 and 2 with human papillomavirus and associated risk factors among asymptomatic women in Ghana. Int J Infect Dis Ther 2018;3:45-51.

10. Bernstein DI, Cardin RD, Smith GA, et al. The R2 non-neuroinvasive HSV-1 vaccine affords protection from genital HSV-2 infections in a guinea pig model. NPJ Vaccines 2020;5:104.

11. Perkins D, Chong H, Irvine B, et al. Genital co-infection with herpes simplex viruses type 1 and 2: comparison of real-time PCR assay and traditional viral isolation methods. J Cell Mol Med 2007;11:581-4.
12. Xu F, Schillinger JA, Sternberg MR, et al. Seroprevalence and coinfection with herpes simplex virus type 1 and type 2 in the United States, 1988-1994. J Infect Dis 2002;185:1019-24

13. Egan K, Hook LM, Naughton A, et al. Herpes simplex virus type 2 trivalent protein vaccine containing glycoproteins C, D and E protects guinea pigs against HSV-1 genital infection. Hum Vaccin Immunother 2020;16:2109-13.

14. Torrone EA, Morrison CS, Chen PL, et al. Prevalence of sexually transmitted infections and bacterial vaginosis among women in sub-Saharan Africa: an individual participant data meta-analysis of 18 HIV prevention studies. PLoS Med 2018;15:e1002511

15. Mehta SD, Nandi D, Agingu W, et al. Vaginal and penile microbiome associations with HSV-2 in women and their male sex partners. J Infect Dis 2020 Aug 21 [Epub]. https://doi.org/10.1093/infdis/jiaa529.

16. Issakwisa HM, Mbwile GR, Mbwanji GF, et al. Seroprevalence of herpes simplex virus type 1 among people living with HIV in Mbeya, Tanzania. BMC Infect Dis 2020;20:577.

17. Dargham SR, Nasrallah GK, Al-Absi ES, et al. Herpes simplex virus type 2 seroprevalence among different national populations of Middle East and North African men. Sex Transm Dis 2018;45:482-7.

18. Kimberlin D. Herpes simplex virus, meningitis and encephalitis in neonates. Herpes 2004;11 Suppl 2:65A-76A. 